\title{
Natural kinds: a new synthesis
}

\author{
(Clases naturales: una nueva sintesis)
}

\author{
Anouk Barberousse ${ }^{1}$, Françoise LongY ${ }^{2}$, Francesca Merlin ${ }^{* 3}$, Stéphanie Ruphy ${ }^{4}$ \\ ${ }^{1}$ University of Sorbonne \\ ${ }^{2}$ University of Strasbourg \\ ${ }^{3}$ CNRS \& Université Paris 1 Panthéon-Sorbonne \\ ${ }^{4}$ Ecole normale supérieure - Université PSL
}

\begin{abstract}
What is a natural kind? This old yet lasting philosophical question has recently received new competing answers (e.g., Chakravartty, 2007; Magnus, 2014; Khalidi, 2013; Slater, 2015; Ereshefsky \& Reydon, 2015). We show that the main ingredients of an encompassing and coherent account of natural kinds are actually on the table, but in need of the right articulation. It is by adopting a non-reductionist, naturalistic and non-conceptualist approach that, in this paper, we elaborate a new synthesis of all these ingredients. Our resulting proposition is a multiple-compartment theory of natural kinds that defines them in purely ontological terms, clearly distinguishes and relates ontological and epistemological issues - more precisely, two grains of ontological descriptions and two grains of explanatory success of natural kinds-, and which sheds light on why natural kinds play an epistemic role both within science and in everyday life.
\end{abstract}

KEYWORDS: Natural Kind; HPC; Essentialism.

RESUMEN: ¿Qué es una clase natural? Esta pregunta filosófica, antigua pero perdurable, ha recibido recientemente nuevas respuestas enfrentadas (e.g., Chakravartty, 2007; Magnus, 2014; Khalidi, 2013; Slater, 2015; Ereshefsky \& Reydon, 2015). En este articulo mostramos que los principales ingredientes para una explicación abarcadora y coherente de las clases naturales están de hecho disponibles, aunque a la espera de una articulación apropiada. Adoptando una perspectiva no reduccionista, naturalista y no conceptualista, elaboramos una nueva sintesis a partir de estos ingredientes. Nuestra propuesta es una teoría de las clases naturales que los define en términos puramente ontológicos, que distingue y relaciona claramente cuestiones ontológicas y epistemológicas - en concreto, dos tipos de descripción ontológica y dos formas de éxito explicativo para las clases naturales-, y que arroja luz sobre por qué las clases naturales desempeñan un papel epistémico tanto en la ciencia como en la vida cotidiana.

PALABRAS CLAVE: Clases naturales; HPC; Esencialismo.

* Correspondence to: Francesca Merlin. Institut d'histoire et de philosophie des sciences et des techniques (IHPST), CNRS \& Université Paris 1 Panthéon-Sorbonne, 13 rue du Four, 75006 Paris (France) - francesca.merlin@univ-paris1.fr - https://orcid.org/0000-0002-2335-3429

How to cite: Barberousse, Anouk; Longy, Françoise; Merlin, Francesca; Ruphy, Stéphanie (2020). "Natural kinds: a new synthesis»; Theoria. An International Journal for Theory, History and Foundations of Science, 35(3), 365-387. (https://doi.org/10.1387/theoria.21066).

Received: 06 Agoust, 2019; Final version: 09 June, 2020.

ISSN 0495-4548 - eISSN 2171-679X / (c) 2020 UPV/EHU 


\section{Introduction}

What is a natural kind $(\mathrm{NK})$ ? Anyone interested today in this old yet lasting philosophical question cannot complain about a lack of fresh insights. In the past twenty years or so, philosophy of science in particular has not been short of various, competing propositions on what it takes for a class to qualify as a NK. These offerings have ranged from strong essentialist accounts such as Wilkerson's (1995) or Ellis' (2001) microphysicalism, to more epistemologically oriented views focusing on explanatory or predictive power (e.g. Laporte, 2004; Dupré, 1993), including the much discussed Homeostatic Property Cluster (HPC) theory developed by Richard Boyd in the 1990's (see Boyd 1991, 1999).

A series of more recent articles ${ }^{1}$ (Magnus, 2014; Slater, 2015; Ereshefsky \& Reydon, 2015 ) contests on good grounds the consensus that "HPC $=\mathrm{NK}$ ", as Magnus puts it, which has been settling in among philosophers of science for the last fifteen years. For these authors, on the contrary, NKs can be of different sorts. Accordingly, they propose new definitions of the NK concept. Each of these propositions gives valuable insights but fails, in the end, to provide a comprehensive enough theory of NKs, i.e., a theory which succeeds in accounting for any sort of NKs and for their role in our successful epistemic practice, both within science and in everyday life. In other words, the main ingredients of an encompassing and coherent account of NKs are now on the table, but in need of the right articulation. As we will discuss in more detail later, these ingredients are, on the one hand, the main philosophical accounts of NKs (microphysicalism, HPC account, historical account, etc.), all aiming at providing a definition of NKs as well as an explanation of their usefulness in our epistemic endeavor; and, on the other hand, the decomposition of the question "What is a NK?" into two different questions, the first about what distinguishes NKs from non-NKs, and the second about the different ways to be a NK.

Our aim in this paper is to elaborate a framework that explicitly integrates these ingredients by shedding light on how they relate to each other. Hopefully, our synthesis will constitute a new basis for further philosophical discussion about NKs. More specifically, its main features are the following: 1) an "ontology-only" theory of NKs where both the answers to the bi-partite question "What is a NK?" are purely ontological; 2) a multiple-compartment theory of NKs that clearly distinguishes and relates ontological and epistemological issues - more precisely, two grains of ontological descriptions of NKs and two grains of explanatory roles of NKs in our successful epistemic practice; 3) a theory of NKs that makes room for both NKs referred to by scientific categories and NKs referred to by vernacular categories because, despite their difference in degree as regards their epistemic usefulness, they both refer to classes characterized by some sort of ontological unity.

We proceed as follows. In section 2, we briefly review the main philosophical accounts of NKs. In section 3, we specify the main features of our own take on the debate. In section 4, by building on Magnus' (2014) splitting of the question "What is a NK?' into two further questions: "What criteria distinguish NKs from arbitrary classes?" and "What features of the world satisfy these criteria?", we distinguish and articulate the ontological and epistemological components of the answers, and argue that epistemological components

1 These papers themselves rely on previous work by Ereshefsky \& Matthen (2005) and Wilson, Barker, \& Brigandt (2007). 
should be removed from a sound definition of NK. Connecting ontological and epistemological components and distinguishing between two grains (general/specific) of these components lead us to a multiple-compartment theory of NKs (Table 1). In section 5, we describe this multiple-compartment theory in detail. Finally, in section 6 , we emphasize the benefits provided by our integrative account, to wit, its ability both to make room for categories used in everyday life and to accommodate scientific change.

\section{Main philosophical accounts of NKs}

At the core of any philosophical account of NKs is the acknowledgment that not all the categories used in natural or scientific languages have the same status vis à vis knowledge-building. It is commonly assumed that some of them, such as "water" or "enzyme", refer to natural groupings, and are thus endowed with properties that make them peculiarly useful within science and everyday life, while others, such as "gift" or "white thing", regarded as arbitrary, are attributed, at most, virtues of a pragmatic nature. For instance, a strong difference is assumed between "being water" and "being a gift", because we can infer a lot from the former, like "boiling at a temperature above $100^{\circ} \mathrm{C}$ when salted", whereas knowing that something is a gift does not enable us to infer much about its properties. Contrary to arbitrary categories, categories like "water" are taken to refer to classes possessing an ontological unity of some sort that accounts for some of their properties, the ones we use in knowledge-related tasks. In other words, these categories are supposed to refer to $\mathrm{NKs}^{2}$. As such, they are endowed with remarkable epistemological features. Since John Stuart Mill (1843) at least, the major epistemological feature associated with NK-categories has been inductive richness, i.e., the capacity of giving rise to many correct, non-trivial, inductive inferences ${ }^{3}$. However, they may demonstrate other features, like appearing in nomological statements ${ }^{4}$.

Let us briefly review the currently most discussed accounts of NKs in contemporary philosophy of science. Putnam (1975) and Kripke (1980) hypothesized that the members of a NK, contrary to those of a mere class, share an essence. According to the essentialist account, NKs are defined by a set of possibly unknown essential properties. What it means to have an essence has received many interpretations since the $1980 \mathrm{~s}$, as Khalidi $(2013$, ch. 1) emphasizes. For most philosophers, NKs defined by an essence do not overlap, have precise delimitations, and do not allow for exceptions (all members of a NK must have all the common properties defining it). The most common form of essentialism is microstructuralism. According to it, a NK is defined by the common microscopic composition shared by all its instances (Ellis, 2001). This composition explains, among other things, the observable properties of the instances used to establish the prima facie classifications. Water and gold are good examples of NKs defined by microstructure because it is commonly sup-

2 We will be careful to use terms as unambiguously as possible. In the paper, "category" will refer to a linguistic or mental device enabling us to refer to groupings of various sorts, as will "concept", "notion", "term" or "classification". "Kind" and "class" will refer to the groups themselves.

3 We follow Khalidi and interpret "non-trivial inferences" as meaning inferences that "not follow as a matter of logic from those properties that we already associate with the kind" (Khalidi, 2013, p. 52).

4 For other epistemological features associated with NK categories, see Ereshevsky \& Reydon (2015). 
posed that being $\mathrm{H}_{2} \mathrm{O}$, or having atomic number 79 , respectively explains why water freezes at $0^{\circ} \mathrm{C}$ and gold is malleable. Nonetheless, the microstructural account has been criticized as unable to make sense of biological species being NKs, whereas cats and oaks commonly count as paradigmatic NKs. The Homeostatic Property Cluster (HPC) account (Boyd, 1991) has thus been proposed to widen the possibilities opened by the microstructural account. According to the HPC account, instances of the same NK need not share microscopic properties; they share instead a cluster of properties enabling them to constitute cohesive wholes. As Kornblith explains, self-maintenance in organisms, i.e., homeostasis, seen as a model that may be applied to NKs, leads to the idea that "a natural kind is a cluster of properties which, when realized together in the same substance, work to maintain and reinforce each other, even in the face of changes in the environment" (Kornblith, 1993, p. 35). Now, because homeostasis allows for imperfect sharing - it explains similarities that are less than perfect - HPC can accommodate kinds such as species of organisms in which many largely shared properties need not be present in every member. But the HPC account, in turn, has been criticized (Millikan, 1999) for letting aside the historical dimension of biological species. Millikan argued for the existence of historical NKs that are grounded in processes warranting historical continuity. Some have also emphasized that certain kinds are best defined by their functions, thereby proposing a functional account of NKs (Putnam, 1975; Boyd, 1999; Weiskopf, 2011; Manafu, 2013). More recently, some have even envisaged mixed types, such as kinds that may have a "physico-functional" nature (e.g., Tobin, 2010).

In spite of these various criticisms, as Ereshefsky \& Reydon (2015, p. 969-970), Magnus (2014, p. 471-472), and Slater (2015, p. 376-377) alike stress, the HPC account is often taken nowadays to be the general theory of NKs, at least once it is interpreted broadly enough so as to accommodate the microstructural NKs as well ${ }^{5}$. However, we share Ereshefsky \& Reydon's, Slater's, and Magnus' diagnosis about the prospects of the HPC account. Even when interpreted broadly, it is still far off the mark. Its main insufficiency is its incapacity to satisfactorily account for a series of apparently legitimate NKs referred to by scientific categories. For instance, some chemical categories, like acids, which are better conceived as referring to functional kinds rather than HPC (Stanford \& Kitcher, 2000; Needham, 2002; Hendry, 2006; Chang, 2012; Manafu, 2013) ${ }^{6}$.

\section{Defining our take on the debate}

Three main general features characterize our own approach to the debate about NKs. First, we specify our naturalistic approach; then, we discuss the relationship between NKs and scientific and vernacular categories respectively; finally, we spell out what to expect from a philosophical theory of NKs as regards its nature (epistemological and/or ontological).

5 This corresponds to the current vulgate about NKs, i.e., the way Boyd's theory has been recently presented (e.g., Samuels \& Ferreira, 2010). However, this does not correspond to the way Boyd himself introduced his account in the 1990s, and still conceives it (oral remark, PSA 2016, Atlanta).

6 For more examples of putative NKs showing the insufficiency of the HPC account, see Ereshefsky \& Reydon (2015, p. 973-977). 


\subsection{Natural Kinds in A NATURAListic FRAMEWORK}

Given the traditional association between NKs and inductive richness, the latter being at its greatest in science, commitment to a naturalist approach naturally follows. Consequently, along with most philosophers currently working on the topic (Magnus, 2014; Slater, 2015; Ereshefsky \& Reydon, 2015; Khalidi, 2013, 2016; Chakravartty, 2007), our inquiry about NKs pertains to scientific or naturalized metaphysics, which is, as Chakravartty says, a metaphysics "inspired and constrained by the output of our best science” (2007, p. 33). To put it briefly: naturalized metaphysics sets itself apart from other approaches, such as descriptive or analytic metaphysics, by insisting on the importance of science within metaphysical inquiry. Naturalized metaphysics today comes in many guises, depending on both how the constraint of accommodating science is understood, and on whether or not it embraces reductionism. It suffices for our purpose to specify that our naturalist approach is not a reductionist one. Along with Chakravartty (2007) and Khalidi (2013), we think that one of the tasks of naturalized metaphysics is to clarify and elaborate the ontology of the various sciences without considering whether or not some sciences can be reduced to others. Accordingly, we do not suppose that the types of entities mentioned within, say, psychology or biology have to be identified with, or reduced to, entities at a more basic ontological level. Such a non-reductionist, naturalist approach may be called "transversal". In our inquiry about NKs we will, therefore, adopt an equal treatment in regards to the various successful and well-established scientific categories within all branches of the natural and social sciences.

Another main general feature of our approach, we call "non-conceptualism", results from the philosophical insights brought by Putnam (1975) and Kripke (1980) about NKs and NK-terms. They explained how an NK-term, such as "water" or "lion", could refer to a class of entities constituting an ontological unity of some sort even if we did not know much about these entities and our conception of them was flawed. More generally, they showed that with NK-terms and concepts, contrary to what happens with other general terms and concepts, the reference is not necessarily an image of the concept, and may differ significantly from the class exactly fitting the descriptive definition of the concept. To sum it up, in the case of NKs, one cannot take concept and reference as two sides of the same coin.

Kripke's and Putnam's way of conceiving of the relationships between language and NKs, which we have just briefly presented, does not assume anything about what NKs are, but it enlightens what the target of a philosophical inquiry about the nature of NKs should be and how it should be carried out. Its target is the reference (or extension) of the NK terms. And the fact that the concept and the reference of a NK-term are not usually reflecting one another has two consequences. First, conceptual analysis is not mandatory to investigate NKs. Second, to avoid possibly damaging ambiguities one has to make clear whether NKs are to be equated with concepts or with classes. For us NKs are classes. So, the perspective we adopt here is significantly different from Chang's (2016), Havstad's (2016), Kendig's (2016), and Nickel's (2015), to name a few of those who adopt a conceptualist approach to NKs. For instance, Chang (2016, p. 33) makes clear from the start that he uses the words "kind" and "natural kind" to designate classificatory concepts rather than classes

7 For an overview of the various trends in naturalized metaphysics, see Chakravartty (2013). 
of entities or properties. Consequently, in this paper, we address questions concerning classes and differences among classes.

\subsection{NKS AND THEIR RELATIONSHIP WITH SCIENTIFIC AND VERNACULAR CATEGORIES}

When reviewing the main philosophical accounts of NKs in contemporary philosophy of science (section 2), we stressed their lack of comprehensiveness, which leads to the more general issue of determining what it takes for a theory of NKs to be comprehensive enough. Should it encompass all the kinds corresponding to all scientific categories or only some of them? Should it also make room for some kinds referred to by vernacular categories? We successively address these two questions.

\subsubsection{NKs and scientific categories}

What does our transversal naturalist approach imply as regards scientific categories? Does it differ from other naturalist approaches? And if so, how? Our commitment to naturalism implies that we want to end up with a theory of NKs according to which our successful scientific categories do refer to NKs. This goal is by no means peculiar. As a matter of fact, most philosophers are willing to endorse it (Wilkerson (1995) and Ellis (2001) might be exceptions). Does a naturalist approach need to put stronger constraints? We claim that it does not and that, moreover, putting additional constraints have undesirable consequences. Let us spell out why by contrasting our naturalist approach with Ereshefsky \& Reydon's. They contend that "natural kinds on [their] account are not necessarily eternal ontological categories; they are foremost grouping picked out by our best scientific theories and classificatory programs" (Ereshefsky \& Reydon, 2015, p. 984). In other words, their naturalist approach constrains the class of NKs to adjust to the set of successful scientific categories. We contend that this constraint is far too restrictive because of at least two reasons.

The first reason goes as follows. Any characterization of scientific categories is bound to have epistemological components since the way in which categories are chosen or devised in science depends in large part on their involvement in a series of epistemic activities (e.g. explaining, providing useful classifications, delineating new lines of research). Accordingly, Ereshefsky \& Reydon (2015) emphasize that scientific categories are supposed to satisfy determinate epistemological requirements such as, e.g., being part of some successful research program. Consequently, deriving the concept of NK from a survey of current successful scientific categories, as Ereshefsky \& Reydon (2015) do, precludes the possibility of defining NKs in purely ontological terms. But this impossibility turns out to have unwanted consequences in a naturalistic framework, as we argue in detail in section 4 .

The second reason why a naturalist approach should not be restrictive at the outset directly follows from the view on the relationship between NKs and non-scientific (or vernacular) categories that we present in the next section. Identifying NKs to the referents of scientific categories amounts to excluding the possibility that some NKs may be referred to by vernacular categories (or even not be referred to at all). As we explain below, this is neither desirable nor tenable. 


\subsubsection{NKs and vernacular categories}

Limiting the range of NKs to the kinds corresponding to scientific categories indeed raises two issues, namely, what is the reference of vernacular terms like "lily" and "cedar", and how to account for historical continuity between scientific and vernacular categories.

"Lily" and "cedar" are natural kind terms of ordinary language that correspond to no scientific category according to contemporary standards. However, the categories of lily and cedar do not appear arbitrary (as does the category of all white objects whose mass range between 5 and $10 \mathrm{~kg}$ ) or void or absurd (as do the categories of phlogiston and witch). As Dupré (1993, ch. 1, 2) makes clear, these categories possess many more features in common with scientific categories than with arbitrary ones. Specifically, they refer to groupings that allow for a significant number of successful inductions. Since there are more differences between an arbitrary class and the class of lilies than between the latter and a taxon featuring in today's botanic classification, we are more justified to consider the class of lilies as a NK than to consider it as a mere class.

Historical continuity is a second reason to resist limiting the range of our analysis to scientific categories. Many categories, e.g., water, gold, cow, etc., that have now become objects of scientific investigations and part of well-established scientific classifications, had a long history before entering science. There is usually no specific moment in history no historical gap in the use of terms, no declaration or debate - that one can point to, at which the change of status of these categories from not-scientific to scientific took place. There appear to be many intermediate stages between being a purely vernacular category that is involved in no scientific research program and being a scientific category. For instance, there seems to be no way to precisely determine when water or cow turned from being purely vernacular categories to being scientific ones, or when croup (the disease) stopped being a medical scientific category to become a merely vernacular one. It is similarly difficult to tell the status of some present-day categories. For instance, is dementia a scientific or a vernacular category? Now, the continuity between vernacular and scientific categories does not fit in well with the claim that a category or general term could change status and shift from referring to a mere class to referring to a NK. Such a shift supposes a semantical jump that does not accord with gradual change. A major advantage of Putnam's and Kripke's approach to the reference of NK terms is precisely to account for such continuity by insisting that discovering the nature of, e.g., water and gold has been a longtime project that progressively became scientific ${ }^{8}$.

\subsection{What to expect from a Philosophical theory of NKs}

From a philosophical theory of NKs one should expect a definition of NKs as well as an explanation of why they are interesting for science in particular (what benefits does science obtain from using natural categories, i.e., categories corresponding to NKs, rather than conventional ones). The task of defining NKs thus consists in identifying what are the features specific to NKs as opposed to mere classes, and which explain the epistemic success of using NKs in science as well as in everyday life.

8 Brigandt (2003) and Griffiths (2004) rely on this view when they claim that NKs may be viewed as investigative kinds, i.e., kinds that are objects of open-ended investigations. 
For some philosophers of science, NKs should be defined purely by epistemological features, that is by the epistemological features displayed by the categories that scientists consider NK-categories. This is the position, for instance, of those who think that NKs "are groups corresponding to categories that best serve our epistemic aims" (Franklin-Hall 2015 , p. 938). In other words, this view corresponds to the idea that we use two different names for classes depending on the role the categories they correspond to play (or not) within science and with respect to our practical interests, even if we do not know whether there is any ontological difference between them ${ }^{9}$. But it is also a position taken for prudence sake by some more realistic-inclined philosophers about NKs. Ereshevsky \& Reydon (2015), for instance, even if they think that NKs have distinctive ontological features ${ }^{10}$, judge it better not committing themselves on such issue.

Such a prudent stance regarding the ontology of NKs has been gaining favor as Boyd's theory has been losing its appeal. Many philosophers have indeed come to the conclusion that Boyd's theory is insufficient to account for the whole range of NKs figuring within science. But rejecting Boyd's theory means losing the well-shared ontological representation of NKs as HPCs, that dominated for at least two decades. Avoiding ontological commitments, if possible, appears therefore a wise move. If we can distinguish NKs from mere classes using only epistemological features, why should we take the risk of relying on hazardous ontological hypotheses? As Lemeire (2018) stresses, this is the position adopted by Magnus (2012) and by Ereshevsky \& Reydon (2015). However, the choice of defining NKs in purely epistemological terms comes with a price. As Lemeire well explains, it means leaving unanswered a major question, that of justifying the proposed criterion by showing that it is in fact doing what it is supposed to do, that is sorting kinds that are in some sense natural. As Lemeire stresses, the fact that there is no obvious agreement on which kinds are natural (for instance, essentialists do not agree with HPC's partisans) demonstrates that "it really is necessary for a successful theory [of NKs] to provide both a set of criteria and a theoretical account of the naturalness of kinds to justify these criteria" (Lemeire, 2018, p. 5). To sum it up, the proposal of a sorting criterion for NKs cannot go without an explication of what it means to be natural and how being natural is related to being epistemologically fruitful. Therefore, every "epistemology-only theory" of NKs, be it general (NKs contribute to scientific success) or specific (NKs contribute to specified epistemic tasks), cannot do the job: one cannot obtain a satisfactory account of NKs without introducing some ontological features.

In this paper, we fully endorse Lemeire's rejection of "epistemology-only theories" of NKs and go a step further. As we show below, NKs should be defined in purely ontological terms.

We have spelled out in this section the three main features of our approach to the debate about NKs. It is now time to explain how we mean to connect our approach to NKs

9 Note however that Franklin-Hall refines this view by admitting that natural kinds are "partially objective", i.e., when they are "invariant across assessors enjoying distinct stances of points-of-view" (Franklin-Hall, 2015, p. 938).

10 Their realist stance shows for instance in the following statement: "[ $t$ ]his criterion elaborates the longstanding view that natural kinds are at least to some extent grounded in nature-that natural kinds are kinds in the empirical world that are to some degree independent of our classificatory practices" (Ereshevsky \& Reydon, 2015, p. 981). 
with the insights that well-established scientific categories nevertheless provide us with about NKs. In order to do so, we will rely on Magnus' (2014) bipartite analysis of the question "What is a NK?" because, as we will show, it allows one to account for the various categories that are taken to be NKs, both within all branches of the natural and social sciences and in everyday life.

\section{Splitting the NK question into two}

In this section, we claim, following Magnus (2014), that the question "What is a NK?" should be decomposed into two questions. After making clear why this decomposition is particularly suited and fruitful in the context of our transversal, naturalist approach, we explain why both questions about NKs should be answered in purely ontological terms. We also specify how answering each question should contribute to a comprehensive account of NKs, which leads us to provide, at the end of the section, a first glimpse of the multi-compartment theory we present in section 5 .

\subsection{Q1 AND Q2}

Magnus (2014, p. 472) argues that the question "What is a NK?" should not elicit one answer but two, because it actually encompasses two different questions that need to be distinguished:

\footnotetext{
"First, what criteria distinguish natural kinds from arbitrary categories? Second, what features of the world make some categories but not others satisfy these criteria?"11
}

We shall call these two questions respectively Q1 and Q2. According to Magnus, Q1 (which he labels the "taxonomy question"), "asks about natural kinds as such" (Magnus, 2014, p. 472): its answer consists in providing a general account of "naturalness"12. As for Q2 (which Magnus labels the "ontology question"), its answer "specifies what it is that satisfies that characterization" (i.e., natural kindhood) "by explaining the different underlying natures of different natural kinds" (Magnus, 2014, p. 472, 473).

Let us now present why we also find it important to distinguish between Q1 and Q2. This is more easily done if we explain beforehand why Q1 and Q2 have not been commonly distinguished in the literature before Magnus' paper. As a matter of fact, the two questions have long been mistaken for one. This is not so surprising, since within the two main accounts of NKs that have been proposed as a general theory, i.e., microstructuralism and HPC, Q1 and Q2, if asked, would not receive two specific answers, different from each other. Rather, they would elicit the same answer, namely "sharing the same microstructure" for the microstructuralist account, and "sharing a relatively stable cluster of properties as

${ }^{11}$ Contrary to us, Magnus does not use "categories" to name only linguistic or mental devices. To follow our convention stated note 2 (section 2), one should replace "category" by "class", which gives the following reformulation: "First, what criteria distinguish natural kinds from arbitrary or mere classes? Second, what features of the world make some classes but not others satisfy these criteria?".

12 In what follows, we will use the expression "natural kindhood" instead of "naturalness". 
the result of a causal mechanism" for the HPC account ${ }^{13}$. Within these accounts, Q1 and Q2 would receive the same answer because each presupposes the existence of just one type of NKs (defined by a microphysical structure and by a relatively stable cluster of properties, respectively). As Magnus explains, both claims: "a NK is a kind whose members have the same microphysical structure" and: "NK = HPC" offer at the same time (1) a suitable answer to Q2 by specifying features of the world that make these classes genuine NKs, and (2) an answer to Q1 as these features can be used as criteria distinguishing NKs from mere classes. Now, a more thorough investigation into the rich variety of successful and well-established categories we observe in all the branches of science obliges the transversal naturalist to abandon these proposals: our scientific categories are too various to be accounted for just by HPC or by microstructure ${ }^{14}$.

Giving up these perspectives on NKs in which Q1 and Q2 are conflated is particularly suited for the philosopher who adopts a transversal viewpoint for the following reason. As suggested by Magnus (2014), it lets open the possibility of providing several answers to Q2, each pointing to some particular underlying ontology of NKs, and an answer to Q1, presenting some common criteria that can sort all the identified types of NKs out from mere classes.

Some might defend that a theory of NKs can, or even should, dispense with addressing $\mathrm{Q} 1$ and settle with several answers to Q2. For instance, one might claim that Q1 cannot be answered because there is no such thing as a common feature of natural kindhood shared by the various types of NKs. However, this view is not tenable because, as a matter of fact, there are already some good candidates for such a feature, as we will show later (section 5). Else, one might claim that identifying such common feature is of no interest to a philosopher of science. But this option is unfortunate because it results giving up the explanatory ambition of the investigation we are pursuing, which is to find a general explanation for the fact that NK categories (i.e., categories that are supposed to refer to NKs) are so useful throughout the sciences and in everyday life. This point will be made in greater details in section 4.4, where we will specify the overall expectations of a philosophical theory of NKs.

\subsection{Why ANSWERs TO Q1 AND Q2 ARE BOTH PURELY ONTOLOGICAL}

Let us consider the sort of philosophical issues $\mathrm{Q} 1$ and $\mathrm{Q} 2$ respectively address. By its very wording, Q2 is a purely ontological question. In fact, as Magnus (2014, p. 472) stresses, it is unambiguously directed towards "features of the world", which is why he calls it the "ontology question". Actually, the most discussed accounts of NKs in contemporary philosophy

${ }^{13}$ It might be argued that Boyd himself implicitly distinguishes Q1 and Q2, since one can split it into two parts, the first answering to Q1 (there is a cluster of properties stably holding together), and the second answering to (these properties hold together in homeostasis by a specific causal mechanism). We thank an anonymous referee for suggesting this reading of Boyd's HPC theory. In section 5 we will show how Q1's and Q2's answers differ in terms of degree of generality, and how they relate to each other.

${ }_{14}$ Taking this variety into account has led some philosophers to waive the very idea of a general theory of NKs. For instance, it has led Hacking $(2007$, p. 203) to claim that "there is no such thing as a natural kind", no such thing as a "well-defined or definable class whose members are all and only natural kinds"; although he had been one of the first philosophers to defend Boyd's account in the 1990s. 
of science provide Q2 with ontological answers: they consist in ontological types, i.e., types only defined by ontological features (microphysical structures, homeostatic mechanisms regulating an array of properties, roles or functions within some determinate context, historical mechanisms of production, etc.). Thus, the ontological nature of answers to Q2 is in line with the major definitions of the NK category proposed within the last fifty years. ${ }^{15}$

Determining the nature of Q1 is not so straightforward. Is it purely ontological too? Or, on the contrary, now that the two questions are no more conflated, shouldn't the criteria distinguishing NKs from mere classes also rely on the role NKs play in knowledge-related tasks, i.e., on the epistemological features we associate with NKs (the most remarkable being inductive richness)? Magnus (2014, p. 472) rejects the option according to which Q1 is a purely epistemological question and thus argues that it would be a mistake "to call" the answers to Q1 and to Q2 "the epistemic and the metaphysical conceptions, respectively” (Magnus, 2014, p. 472). We agree with Magnus and offer another reason ${ }^{16}$, namely, the context-dependency of the role of NKs in our epistemic practice.

\subsubsection{The context-dependency of the role of $N K$ s in our epistemic practice}

Let us start by establishing that the answer to Q1 cannot be purely epistemological by taking into account possible changes in scientific practices.

Consider the following fictitious example about inert gases (the discovery history is invented). Imagine that, during a certain period of time, the answer to Q2 "What is an inert gas?" was the list "helium, neon, argon, krypton", each identified by its atomic number (i.e., an ontological answer). Suppose now that the four gases had all been discovered by the same scientist using a particular apparatus X of her invention. During that period, the epistemological property "being discovered by showing feature $F$ within apparatus $X$ " and the ontological disjunctive one "being either of atomic number 2, 10, 18 or 36" would have appeared co-extensional. Suppose now that, at the end of this period, xenon is discovered by using another apparatus Z; as a consequence, Q1's and Q2's answers would no longer be consistent. On the contrary, if the answer to Q1 had been based on the ontological property "having its outermost electron shell full" - which is directly related to the chemical properties of inert gases - the two answers would still be co-extensional, thus consistent. In fact, knowing, thanks to the chemical theory, why being-inert results from having-itsoutermost-electron-shell-full justifies associating this particular ontological criterion (Q1's answer) to what-is-to-be-an-inert-gas (Q2's answer).

Now that we have shown why the answer Q1 cannot be purely epistemological, let us consider the two remaining options: a) the criterion distinguishing NKs from mere classes only includes ontological features; b) it both includes ontological and epistemological features. Magnus seems to choose option b) when he claims that one might answer Q1 "by characterizing the role that natural kinds necessarily play in our account of the world"

15 This confirms that a general theory of NKs cannot be purely epistemological (see our discussion of Lemeire (2018) in section 3.3). But the nature of Q1, epistemological and/or ontological, remains an open question.

${ }_{16}$ Magnus' argument is one of epistemic inaccessibility. By assuming that epistemic properties have to be epistemically accessible, he argues that we may fail to discover the criteria which distinguish NKs from mere classes, even if one exists. 
(Magnus, 2014, p. 472). However, option b) is unsatisfactory. We show it by drawing again on the context-dependency of the role of $\mathrm{NKs}$ in our epistemic practice. Here is the argument: a NK from the point of view of Q2, namely, an instance of a particular type of NK defined by purely ontological features, may fail to be a NK from the point of view of Q1 whenever it fails to play a determinate epistemic role. For instance, let us imagine that there are some ontological types of NKs in the world (i.e., classes possessing an ontological unity of some sort) that we have no idea about, because our epistemic interests have not led us (until now, at least) to carve nature at these joints. Now, from the point of view of Q2, such classes are NKs because of this unity they have that mere classes do not have. However, if the criteria for distinguishing NKs from mere classes also required some epistemic role for NKs (like to support successful inductions), these classes would not be NKs from the point of view of Q1 because of our ignorance with respect to their existence.

So, because of the context-dependency of the role of NKs in our epistemic practice, Q1 and Q2 are best conceived of as both bearing purely on ontology, with Q1 asking for a low-resolution answer, and Q2 requiring a higher resolution answer, as will be explained later (see section 5$)^{17}$.

Up to now, we have argued for a purely ontological definition of NKs. For clarificatory purpose, we will now situate ourselves among various readings of Boyd's accommodation thesis, thereby strengthening our claim that dependency on the epistemic context is compatible with an ontological take on NKs.

\subsubsection{Boyd's accommodation thesis: a clarification}

It is currently disputed whether Boyd's HPC account of NKs is purely ontological or not. For instance, Ereshefsky \& Reydon (2015) argue that a good understanding of Boyd's work compels us to break up with this classical view of NKs as ontological features of the world, in particular once we take into account the open-endedness of science, and the resulting context-dependence of successful scientific categories. More precisely, they argue that HPC theory "moves away from the traditional idea that the kinds used in the sciences are natural kinds in the sense of their entirely being objective features of the world out there, toward a view of natural kinds as products of the interplay between human classificatory interests and states of affairs in nature that determines the boundaries of kinds. This is Boyd's 'accommodation thesis"” (Ereshefsky \& Reydon, 2015, p. 971).

This reading of Boyd's accommodation thesis, which makes the classificatory interests of humans determine the boundaries of kinds, is debatable. But first, let us present the accommodation thesis by a frequently used quote: "successful induction and explanation always require that we accommodate our categories to the causal structure of the world" (Boyd, 1991, p. 139). Now, this assertion can be read in a way that fits in well with a realist stance considering the NK category as being purely ontological, as do Bird \& Hawley (2011) and Khalidi (2016). Or it can be read as Franklin-Hall (2015) and Massimi (2014) do. For

17 Surprisingly, this way of conceiving of the relation between Q1 and Q2 it also suggested by Magnus when he says that the difference between the two questions is "one of specificity and depth" (Magnus, 2014, p. 473). Such a difference requires in fact that answers to both are similarly ontological, the answers to Q2 being only more detailed and specific than the answer to Q1. See section 5 for an elucidation of the relation between Q1 and Q2 that meets Magnus' intuition. 
instance, Franklin-Hall (2015, p. 929) claims, while referring to Boyd's accommodation thesis, that "the view is anti-realist in virtue of making the natural kinds depend on our scientific projects and practices". We disagree with her and argue that the accommodation thesis turns out to be perfectly compatible with realism if, instead of being understood as "making natural kinds depend on our scientific projects and practices", it is understood as "making the natural kinds highlighted by science depend on our scientific projects and practices", in accordance with one of the readings proposed by Khalidi (2016, p. 410). In other words, Boyd's accommodation thesis can be read as saying either that human interests determine the natural kinds themselves or that they determine which ones to focus on. Khalidi makes plain that the latter reading has a consequence that may appear repellent to some, but that he himself is disposed to accept, the consequence "that there may be more kinds of things in the universe than are dreamt of in a completed science" (Khalidi, 2016, p. 414).

The more general lesson we draw from Khalidi's interpretation of the accommodation thesis is the following: scientific change can be taken into account without necessarily introducing epistemological elements within the definition of NKs. The epistemic context, at any given time, just tells us which NKs we should focus on.

\subsection{What is EXPECTED From ANSWERING Q1 AND Q2}

\subsubsection{An answer to Q1 should offer more than a criterion}

Similarly to Magnus, Slater (2015) distinguishes two questions regarding the nature of NKs; but, unlike Magnus, Slater focuses on the epistemic practices that the answers we give them should help explain, as clearly appears in the following quote where they are introduced:

\footnotetext{
"One question concerns the interpretation of the phenomena gestured at using such metaphors as 'clustering', 'holding together', 'sociability/intimacy', and how exactly such phenomena contribute to our epistemic projects. A second question asks after the metaphysical explanation for these phenomena. Although the questions blur together to some extent, we might think of the first question as directed at articulating a particular explanandum (the existence of a clustering phenomenon - or phenomena- and its connection to some of our epistemic practices) and the second as its explanans: what accounts for the clustering/sociability of properties in virtue of which we enjoy a measure of inductive success." (Slater, 2015, p. 382; our italics)
}

Even if Slater's two questions are not exactly the same as Magnus', their targets are the same. The first question aims at identifying an ontological structure that would be common to and distinctive of all NKs, while the second aims at explaining the existence of this common structure by highlighting what produces it. The most perceptible difference between Slater's and Magnus' positions concerns the first question. While Magnus simply aims at a criterion, Slater aims at identifying the minimal ontological structure that can explain the epistemological usefulness of referring to NKs through all sciences, as he forcefully says in the following quote:

"I suggest that an account of natural kinds would do better to focus on the special sort of stability a cluster of properties might possess in virtue of which it is apt for induction and explanation rather than focusing on the something causing that stability." (Slater, 2015, p. 396; our italics) 
Putting together Magnus' and Slater's claims helps us understand what can be expected from answering Q1, besides finding out a criterion sorting out NKs from non-NKs. As we have seen, a general theory of NKs is expected to elucidate the epistemological usefulness of referring to NKs within and outside science. Now, as Q2 elicits several answers pointing to different ontological types of NKs, none of these answers can provide us with a general explanation of the epistemological usefulness of NKs. In line with Slater's suggestion, we argue that an answer to Q1 can offer such a general explanation: identifying the ontological feature that makes NKs epistemologically useful amounts to identifying a property (or a set of properties) ${ }^{18}$ that both sorts out NKs from mere classes and explains why we have categories targeting NKs in many sciences. Hence, a good answer to Q1 should provide us with both a criterion of natural kindhood and an explanation of why references to NKs are both ubiquitous in science and in everyday life discourses.

\subsubsection{How answers to $Q 2$ contribute to a theory of $N K s$}

Recall that before Magnus and Slater separated Q1 from Q2, the question "What is a NK?" was supposed to elicit a single answer that could offer both an elucidation of the ontological nature of NKs, and an explanation of their epistemological usefulness (within and outside science). Now that Q1 and Q2 are distinguished, we have to specify how the answers to each question contribute to a general theory of NKs. As regards answers to Q2, Magnus (2014) and Slater (2015) have both stressed their ontological significance. Neither of them, however, says what their role can be in explaining the epistemological usefulness of NKs, or even if they have any. In the way he articulates the two questions, Slater even seems to suggest that the answer to Q1 offers all that is needed in this regard (Slater, 2015, p. 382). We claim that this is a shortcoming of Slater's and Magnus' proposals: answers to Q2 should also offer accounts of the capacity of NKs to facilitate our epistemic endeavors, albeit, as we will explain later, more specific ones than the answer to Q1.

\subsection{OVERALl EXPECTATIONS FOR A PHILOSOPHICAL THEORY OF NKs}

Summing up the points made in sections 4.2 and 4.3, we argue that a philosophical account of NKs should meet the following expectations: First, it should offer a theory of natural kindhood, namely, an answer to Q1 that both provides a criterion of demarcation between NKs and mere classes, and a general explanation of why we are better off, in our epistemic endeavors, when we deal with NKs than when we deal with mere classes. Second, it should also contain finer-grained elements, in accordance with the ongoing philosophical discussion on NKs that analyzes scientific and everyday practices and mainly focuses on microstructural kinds, HPCs, or other specific types of NKs. Third, it should also show how these finer-grained structures (the types of NKs discovered so far) account for specific forms of successful epistemic endeavors, that is, specific forms of projections, predictions, and extrapolations that are relied on in the various sciences (more on that in section 5).

${ }_{18}$ We do not exclude beforehand that there might be several such features, even though as we shall see, one proposal already present in the literature seems well suited for this function. 
To sum up, these expectations lead us to clearly distinguish and articulate two grains of ontological descriptions (answers to Q1 and Q2), and two grains of explanatory roles of NKs in our successful epistemic practice in science and in everyday life. In the following section, we flesh out this multiple-compartment theory, represented in Table 1.

Table 1. Our synthesis

\begin{tabular}{|c|c|c|c|c|}
\hline $\begin{array}{l}\text { The } \\
\text { question and }\end{array}$ & The ANSWER & \multicolumn{3}{|c|}{ What is expected from the answer? } \\
\hline $\begin{array}{c}\text { What is a } \\
\text { NK? }\end{array}$ & (is) ontological & $\begin{array}{l}\text { A contribution } \\
\text { to the ontological } \\
\text { image of the world }\end{array}$ & $\begin{array}{c}\text { An explanation of } \\
\text { natural categories' } \\
\text { epistemological features }\end{array}$ & Criterion \\
\hline $\begin{array}{c}\text { Q1 } \\
\text { GENERAL }\end{array}$ & $\begin{array}{l}\text { Stability of properties' } \\
\text { clusters (or some other } \\
\text { property or set of) }\end{array}$ & $\begin{array}{c}\text { YES } \\
\text { In general } \\
\text { descriptions }\end{array}$ & $\begin{array}{l}\text { Inductive richness (or } \\
\text { some other general } \\
\text { epistemological fea- } \\
\text { ture or set of) }\end{array}$ & $\begin{array}{l}\text { YES } \\
\text { (criterion of } \\
\text { kindhood) }\end{array}$ \\
\hline $\begin{array}{c}\text { Q2 } \\
\text { SPECIFIC }\end{array}$ & $\begin{array}{l}\text { Microstructural kinds, } \\
\text { HPCs, Historical kinds, } \\
\text { Functional kinds... }\end{array}$ & $\begin{array}{c}\text { YES } \\
\text { In specific } \\
\text { descriptions }\end{array}$ & $\begin{array}{l}\text { Specific inductive } \\
\text { richness. } \\
\text { Epistemological spe- } \\
\text { cific features depend- } \\
\text { ing on the sort of NK }\end{array}$ & NO \\
\hline
\end{tabular}

\section{The resulting picture: a multiple-compartment theory}

In this section, we describe in details the general architecture of the conceptual framework we propose for the study of NKs, and explain, in particular, how the answers to Q1 and Q2 relate and how they explain the rich epistemic use one can make of NKs in science and everyday life.

The conceptual framework we argue for is presented in Table 1. The table is divided into two main lines according to the above-presented distinction between question $\mathrm{Q} 1$ (What criterion distinguishes NKs from mere classes?) and question Q2 (What features of the world make some classes but not others satisfy this criterion?). In the left-most column, we have indicated how we conceive of this distinction, as made clear in section 4 . The range of Q1 is general. Its object is to identify an ontological structure common to all NKs and distinctive of them. It requires therefore an answer that encompasses all types of NKs identified so far, whereas Q2 asks for specific types of kinds, and requires thereby finer-grained, or higher-resolution, answers.

The next column presents answers to Q1 and Q2. Even if several answers have been proposed to Q1 (more on that later), we choose one for simplicity sake (see below section 5.1). According to it, NKs can be distinguished from mere classes by binding together a set of properties in a distinctively stable way, as we discuss below. As answers to Q2, we put forward the four major types of NKs that science-based philosophical inquiry has iden- 
tified so far, namely, kinds defined by their microstructure, HPC kinds, historical kinds, and functional kinds. We will argue below that these four types realize the distinctive ontological feature of stability that defines NKs in the most general way.

\subsection{THE ONTOLOGICAL PICTURE: STABILITY AND ITS MULTIPLE REALIZABILITY}

Only a few philosophers have bothered to define NKs at a more general level than the one that is specific of answers to Q2. As we have made clear in the beginning of the paper, the main reason for this is that most philosophers have been sharing the view that the HPC account, broadly construed, is the right one, i.e., that it can encompass all characteristic features of NKs. Chakravartty, Khalidi and Slater have, however, proposed a characterization of NKs that is general enough to be an answer to Q1. Chakravartty (2007), searching for the basic transversal ontological categories that science relies upon, identifies kindhood with "sociability between properties". Khalidi (2013), after having surveyed the various types of NKs one may find in the sciences, defines kindhood as what constitutes "a node in a causal network". Slater (2015) suggests, as we have seen, to equate a NK with a "stable property cluster" (SPC). All three proposals are close to one another, as they point to the existence of a strong correlation between a set of properties, be it causal or not, that ensures a stability of some sort.

It is not the right place here to discuss the differences among these three proposals, since they have no relevance for the general architecture we suggest here. Any of these three proposals fits in perfectly well with this architecture. Let us therefore, for the sake of simplicity, stick to Slater's SPC theory and take stability as the common ontological feature that constitutes the answer to Q $1^{19}$. As stated above, we conceive of answers to Q2 as identifying the different types of NKs that realize this shared feature. Micro-structural kinds realize stability because of quantum and electronic relations; HPC realize stability because of a variety of mechanisms regulating a set of properties; historical kinds, among which some philosophers put biological kinds, realize stability because of copying mechanisms that produce reproductively established families; and functional kinds realize stability by having their members designed, naturally or intentionally, to fulfill a determinate causal role. Moreover, note that other types of NKs than those discussed until now in the literature may be discovered in the future: they could then be shown to realize the general feature of stability in other possible, still unknown, different ways.

\subsection{THE RELATIONSHIPS BETWEEN ONTOLOGICAL AND EPISTEMOLOGICAL COMPONENTS}

The next threefold column in Table 1 summarizes the three sorts of insights one can expect from sound answers to Q1 and Q2.

First, it is expected that an answer to the general question: "What is a NK?" contributes to our ontological image of the world, that is, to our knowledge of the world's furniture. As a matter of fact, both answers to Q1 and Q2 do so, each at its own level of generality. The answer to Q1 puts forward the existence of a general property in nature, stability,

19 The difference between Slater's account and ours will be the topic of section 5.4. 
that provides the notion of kindhood (as independent from our minds) with a distinctive meaning, whereas answers to Q2 exhibit different ways this general property may be realized in nature.

Second, we expect that an answer to the general question: "What is a NK?" explains why our categories are epistemically useful when they correspond to NKs. This expectation concerns answers to both Q1 and Q2. As has already been stressed, being inductively rich, that is, allowing for generalizations, extrapolations, and projections, is the major property that makes NK categories useful throughout the sciences. One should not exclude however that their usefulness might also be related to other general epistemological properties, independent, at least in part, from inductive richness. If so, these properties would have to be put next to "inductive richness", as indicated in Table 1. Yet, for simplicity sake, we assume that there is a fundamental property, inductive richness, that is the mark of NKs throughout the sciences and in everyday life, and that any other epistemological feature of NK categories is somehow related to it.

The answer to Q1 is meant to explain the inductive richness of NKs categories by identifying the minimal ontological condition $\mathrm{X}$ that is responsible thereof. In other words, $\mathrm{X}$ has to be such as to support the following claim: it is because all NKs have in common the ontological feature $\mathrm{X}$ that a large series of non-trivial inductions made using NK categories are true. Following Slater, we expect from the framework we put forward that it explains how exactly the phenomena gestured at using metaphors such as "clustering", "holding together", and "sociability/intimacy" contribute to our epistemic projects. We follow him in claiming that "the special sort of stability a cluster of properties might possess" is exactly that "in virtue of which it is apt for induction and explanation" (Slater, 2015, p. 396).

At the Q2 level, the same relation obtains between the components of the ontological image of the world and the way we can rely on them in our epistemic practice, be it in science or in everyday life. Relying on micro-structural kinds allows for explanations based on microscopic components and their relationships, whilst HPC-kinds allow for explanations by homeostatic mechanisms, etc. As stressed above, both levels (both lines in Table 1) are important in regards to the clarification of the relationships between ontological and epistemological features. At the Q1 level, assuming that a category (be it used in science or in everyday life) refers to a stable property cluster only justifies the unspecific claim that there is a set of properties about which non-trivial inductive inferences would be successful were they stated. Going to the Q2-level in order to specify the type of NK concerned in a specific investigation helps reduce the range of projectable properties one may refer to. For instance, assuming that the NK referred to is of the micro-structural type justifies projecting physical and chemical properties, while assuming that it is of the historical type justifies projecting historical patterns and developmental paths. This makes clear why both a general notion of kindhood and the identification of different types of NKs are important to account for our epistemic practices. The answer to Q1 provides us with a generic explanation of why a significant number of inductions involving categories referring to NKs have succeed: namely, because the involved properties are stably clustered. The answers to Q2, in turn, explain the success of one type of inductions as opposed to other types, depending on the type of NKs envisaged. Toulmin (1961) gives an enlightening example in this regard. Chaldeans, he remarks, were keen on making inductions about the periodical return of whatever sort of phenomenon or situation. But, while this type of induction demon- 
strated successful relative to celestial bodies' motions or physical phenomena such as tides, it failed relative to diseases or agricultural calamities. It is only by specifying the causal basis of those phenomena - the types of properties from which they depend- hence the ontological nature of the involved NKs, that one can explain this differential success.

The answers to Q2 may also explain the specificity of generalizations used in the special sciences. For instance, when dealing with historical kinds in biology, we should not expect that they figure in universal generalizations (i.e., laws) but in contingent ones. Beatty, in his 1995 paper regarding what he calls the "evolutionary contingency thesis", argues that distinctively biological generalizations describe historical evolutionary outcomes; and thus that there are no laws in biology, only generalizations that are contingently true (they depend on evolutionary history). If Beatty (1995) is right, assuming that a NK is of the historical type would in fact tell us why we can just formulate these sorts of contingent generalizations about it, contrary to what would be the case if it were a microstructural NK.

Moreover, there are epistemological practices that involve NKs, but which nonetheless cannot be explained or justified unless a finer-grained account of NKs is provided than the answer to Q1. For instance, the fact that chemical elements are microstructural NKs explains the success of the research program launched in chemistry in the Eighteenth Century by Lavoisier and others, which relied on the principle of mass conservation, and aimed to identify quantitative laws (stoichiometry). As a matter fact, the microstructural nature of chemical elements explains both why the assumptions made at the time were correct (e.g. "chemical elements can combine with one another without losing their identity" and the principle according to which "we must always suppose an exact equality or equation between the principles of the body examined and those of the products of its analysis" (Lavoisier, 1789/1960, p. 124)), and, as a consequence, why relying on them gave rise to successful scientific endeavour. In other words, the answers to Q2 complement the answer to $\mathrm{Q} 1$ as regards the explanation of inductive success and help explain the success of other epistemological practices: those that do not just assume the existence of stable property clusters, but require certain sorts of stable property clusters.

Finally, the last column of Table 1 indicates that a sound answer to Q1 does provide a criterion for sorting out NKs from mere classes, whereas answers to Q2 do not provide such a criterion since answers to Q2, being science-based, are open-ended.

\subsection{Are Answers to Q1 AND Q2 both a POSTERIORI?}

A last precision might be in order on how one gets answers to Q1 and Q2, respectively. We have emphasized earlier that the answers to Q2 depend on the advancement of the different scientific disciplines. They point to an open-ended list of features of the world that are discovered by analyzing the successful categories and classifications used in current sciences and in everyday life. In that sense they are a posteriori answers. What about answers to Q1 ? Our main point is that Q1 need not be and should not be answered by generalizing from answers to Q2, and thus is not similarly a posteriori. How then can one get the answer to Q1?

Let us first recall that the answer to Q1 should provide more than a criterion that gives us the means to discriminate NKs from non-NKs. It should also provide us with an explanation of the usefulness of NKs in science and everyday life. How can this ontological feature be identified, if it cannot be obtained by empirical inquiry followed by gener- 
alization? Given the explanatory relationships between the ontological and epistemological components of our theory of NKs discussed above, the answer to Q1 can be reached through a typically a priori sort of philosophical reflection, namely a sort of Kantian transcendental deduction: given the list of epistemological properties displayed by NKs, what ontological feature must NKs have that makes it possible for them to have these very properties? As a matter of fact, the answer to Q1 is not purely a priori, since the list of epistemological properties associated to NKs depends largely on how science categorizes and uses its categorizations to increase knowledge, but it still can involve a significant a priori part. In other words, a response to Q1 needs not be obtained inductively by looking at all the types of NKs individuated so far and searching for whatever common feature they might have ${ }^{20}$.

\subsection{AsSESSING OUR PROPOSAL With RESPECT to SLATER'S}

In so far as we take stability as the ontological feature distinguishing NKs from mere classes, the difference between our proposal about NKs and Slater's SPC theory could sound minimal. Let us briefly explain why it is not the case. First, we endorse an "ontology-only theory" of NKs whereas Slater's SPC theory explicitly incorporates epistemological features into the definition of NKs, when for instance he claims that "the interests and norms of a domain-even a particular research project- (...) influence whether a certain category counts as a natural kind" (Slater, 2015, p. 405): NKs "are genuine features of the world for the relevant domains" (Slater, 2015, p. 407, our italics). Second, we do not propose again another definition of NKs but show how existing accounts can be brought together and articulated with each other within an encompassing two-level theory of NKs. More explicitly, our synthesis clearly distinguishes the ontological aspects of the theory from their implications as regards natural categories' epistemological features, both at the level of Q1 and Q2. In particular, a specific contribution of our account, which other accounts do not make, is that Q2's answers (being it microstructuralism, HPCs, historical kinds, etc.) explain specific epistemological features of NKs in special sciences.

\section{Concluding remarks}

In this paper, we began by reviewing the main philosophical accounts of NKs and by spelling out the main general features of our own take on debate about NKs, namely a transversal, naturalistic and non-conceptualistic approach allowing both scientific and vernacular categories to refer to NKs. Then, following Magnus (2014), we decomposed the question "What is a NK?" into two questions: Q1) What criteria distinguish NKs from arbitrary of mere classes? and Q2) What features of the world make some classes but not others satisfy these criteria? We specified how answering each question should contribute to a comprehensive account of NKs. More precisely, we argued that the answers to these two questions

${ }^{20}$ Note that the answer to Q2 could also be reached through the same kind of a priori philosophical reflection but at a lower level of generality. 
should be purely ontological, in particular because of the context-dependency of the role of NKs in our epistemic practices. Our multi-compartment theory was then ready to be introduced and explained. It distinguishes and articulates two grains of ontological descriptions of NKs (answers to Q1 and Q2, namely, an ontological feature common to all NKs and different particular ontologies realizing it), and two grains of explanatory roles of NKs in our successful epistemic practices (their general inductive richness and types of inductions and generalizations NKs allow to make in specific domains of investigation). We argued that, without introducing any epistemological element in the answers to Q1 and Q2, our "ontology-only" theory still provides an explanation of the explanatory roles of NKs, at different grains, and of why referring to them is useful, and so ubiquitous, in our epistemic practices. The explanation resides in the ontological feature common to all NKs (Q1's answer, i.e., stability), which is the minimal ontological condition of the inductive success of NKs, and in the different ontological types of NKs (Q2's answer, i.e., micro-physicalism, HPC, etc.), which explain NKs' epistemic success at a finer grained level.

As a conclusion, we would like to underline what seems to us the three main benefits provided by our multiple-compartment account of NKs.

First, by proposing that both questions, Q1 and Q2, about NKs should be answered in purely ontological terms, we are in favor of a purely ontological definition of NKs. We thus do not close the door to the possibility of vernacular categories referring to NKs, and do not introduce problematic gaps between scientific categories and categories used in everyday life (section 3.2). This can be contrasted with other recent accounts, in particular Ereshefsky \& Reydon's: contrary to what they claim (Ereshefsky \& Reydon, 2015, p. 983984), the epistemological conditions they put on being a NK are not compatible with the possibility of NKs outside of science, which makes their account prone to inconsistencies. Our purely ontological characterization of NKs that makes room for both scientific and vernacular categories allows our transversal, naturalized approach to escape this kind of inconsistency. We acknowledge a difference in degree between NKs referred to by scientific categories and NKs referred to by vernacular categories as regards inductive richness, science being our most powerful and reliable way to epistemically access the world. For all that, of course, not all vernacular categories can be interpreted as referring to NKs. Some of them (e.g., horoscopic categories such as gemini or aquarius) cannot, since they do not possess any ontological unity. That is, they do not bind together any set of properties in a distinctively stable way; this is why they have no inductive richness at all. By contrast, those vernacular categories (e.g., the gardener's category of lilies or the cook's category of fish) that reveal to be liable to support a series of inductions, even within a limited range, can be considered as referring to NKs; they possess some form of ontological unity accounting for their (limited) inductive richness.

Second, our proposal can accommodate scientific change - new types of NK may appear in future science - as well as philosophical change. Not only may philosophers have not yet identified every sort of NKs referred to in science (see for instance Tobin (2010) for a recent investigation about what sort of NKs proteins might be), but they may also, in the future, find our present characterizations too rough, and decide therefore to divide our present types in more finely defined ones (for instance, by distinguishing various sorts of microstructural kinds).

Third, by combining the two grains of ontological questions, Q1 and Q2, with an unambiguous distinction between ontological and epistemological issues (see Table 1), we of- 
fer a clear account of what a philosophical theory of NKs is meant to explain, and how it should do so. Our multiple-compartment account does build directly on Magnus' and Slater's important insights; but by overcoming some ambiguities, it draws the relevant consequences of their distinction between $\mathrm{Q} 1$ and Q2 for a theory of NKs, and provides a new layout allowing to combine them in a coherent way. More generally, the rearrangement that we propose of various elements of the current philosophical debate on NKs allows us to integrate them in a common framework and to dissipate some ill-conceived lines of opposition (e.g., NKs as HPCs vs. NKs as scientific kinds). The new synthesis we offer hopefully constitutes a more coherent and comprehensive basis for further philosophical discussion regarding $\mathrm{NKs}$.

\section{REFERENCES}

Beatty, J. (1995). The evolutionary contingency thesis. In G. Wolters \& G. Lennox (eds.) Concepts, Theories, and Rationality in the Biological Sciences. The Second Pittsurgh-Konstanz Colloquium in the Philosophy of Science (pp. 45-81). Pittsburgh: University of Pittsburgh Press.

Boyd, R. (1991). Realism, anti-foundationalism and the enthusiasm for natural kinds. Philosophical Studies: An International Journal for Philosophy in the Analytic Tradition, 61(1/2), 127-148.

Boyd, R. (1999). Homeostasis, species, and higher taxa. Species: New interdisciplinary essays. In R. Wilson (ed.) Species: New Interdisciplinary Essays (pp. 141-185). Cambridge, MA: MIT Press.

Brigandt, I. (2003). Species pluralism does not imply species eliminativism. Philosophy of Science, 70(5), 1305-1316.

Chakravartty, A. (2007). A metaphysics for scientific Realism: Knowing the unobservable. Cambridge: Cambridge University Press.

Chakravartty, A. (2013). On the prospects of naturalized metaphysics. In D. Ross, J. Ladyman \& H. Kincaid (eds.) Scientific Metaphysics (pp. 27-50). Oxford: Oxford University Press.

Chang, H. (2012). Acidity: The persistence of the everyday in the scientific. Philosophy of Science, 79(5), 690700.

Chang, H. (2015). The rising of chemical natural kinds through epistemic iteration. In C. Kendig (Ed.) Natural kinds and classification in scientific practice (pp. 33-47). London: Routledge.

Dupré, J. (1993). The Disorder of Things: Metaphysical Foundations of the Disunity of Science. Cambridge, MA: Harvard University Press.

Ellis, B. (2001). Scientific Essentialism. Cambridge: Cambridge University Press.

Ereshefsky, M., \& Matthen, M. (2005). Taxonomy, polymorphism, and history: An introduction to population structure theory. Philosophy of Science, 72(1), 1-21.

Ereshefsky, M., \& Reydon, T. A. (2015). Scientific kinds. Philosophical Studies, 172(4), 969-986.

Franklin-Hall, L. R. (2015). Natural kinds as categorical bottlenecks. Philosophical Studies, 172(4), 925-948.

Griffiths, P. E. (2004). Emotions as natural and normative kinds. Philosophy of science, 71(5), 901-911.

Hacking, I. (2007). Natural kinds: Rosy dawn, scholastic twilight. Royal Institute of Philosophy Supplements, 61, 203-239.

Hawley, K., \& Bird, A. (2011). What are natural kinds?. Philosophical Perspectives, 25, 205-221.

Havstad, J.C. (2016). Protein tokes, types, and taxa. In C. Kendig (ed.) Natural kinds and classification in scientific practice (pp. 74-86). London: Routledge.

Hendry, R. F. (2006). Elements, compounds, and other chemical kinds. Philosophy of science, 73(5), 864875.

Kendig, C. (2016). Homologizing as kinding. In C. Kendig (ed.) Natural kinds and classification in scientific practice (pp. 106-127). London: Routledge. 
Khalidi, M. A. (2013). Natural categories and human kinds: Classification in the natural and social sciences. Cambridge: Cambridge University Press.

Khalidi, M. A. (2016). Natural Kinds. In P. Humphreys (ed.) The Oxford Handbook of Philosophy of Science (pp. 397-416). Oxford: Oxford University Press.

Kornblith, H. (1993). Inductive Inference and Its Natural Ground. Cambridge, MA: MIT Press.

Kripke, S. (1980). Naming and Necessity. Oxford: Basil Blackwell.

Laporte, J. (2004). Natural Kinds and Conceptual Change. Cambridge: Cambridge University Press.

Lavoisier, A. (1789). Traité Elémentaire de Chimie. Translation as given in J. R. Riddick Partington (1960) A Short History of Chemistry. Third Edition. New York: Harper \& Row.

Lemeire, O. (2018). No purely epistemic theory can account for the naturalness of kinds. Synthese, 1-19. https://doi.org/10.1007/s11229-018-1806-8

Magnus, P. D. (2012). Scientific Enquiry and Natural Kinds. From Planets to Mallards. London: Palgrave MacMillan.

Magnus, P. D. (2014). NK $\neq$ HPC. The Philosophical Quarterly, 64(256), 471-477.

Manafu, A. (2013). Concepts of Emergence in Chemistry. In J. P. Llored (Ed.) Philosophy of Chemistry: Practices, Methodologies and Concepts (pp. 659-675). Newcastle: Cambridge Scholars Publishing.

Massimi, M. (2014). Natural kinds and naturalised Kantianism. Noûs, 48(3), 416-449.

Mill, J.S. (1843). A System of Logic, Ratiocinative and Inductive. London: Harrison \& Co.

Millikan, R. G. (1999). Historical kinds and the "special sciences". Philosophical Studies, 95(1), 45-65.

Needham, P. (2002). The discovery that water is H2O. International Studies in the Philosphy of Science, 16(3), 205-226.

Nickel, B. (2015). Explanatory strategies in linguistic practice. In C. Kendig (Ed.) Natural kinds and classification in scientific practice (pp. 37-52). London: Routledge.

Putnam, H. (1975). The Meaning of "Meaning". Language, mind, and knowledge. Minnesota Studies in the Philosophy of Science 7, 131-193.

Quine, W.V., (1970). Natural Kinds. In N. Rescher et al. (eds.), Essays in Honour of Carl G. Hempel (pp. 1-23). Dordrecht: Springer.

Samuels, R., \& Ferreira, M. (2010). Why don't concepts constitute a natural kind?. Behavioral and Brain Sciences, 33(2-3), 222-223.

Slater, M. H. (2015). Natural kindness. The British Journal for the Philosophy of Science, 66(2), 375-411.

Stanford, P. K., \& Kitcher, P. (2000). Refining the causal theory of reference for natural kind terms. Philosophical Studies, 97(1), 97-127.

Stuart Mill, J. (1843). A System of Logic, Ratiocinative And Inductive. Honolulu: University Press of the Pacific, 2002.

Tobin, E. (2010). Microstructuralism and macromolecules: the case of moonlighting proteins. Foundations of Chemistry, 12(1), 41-54.

Toulmin, S. E. (1961). Foresight and understanding: An enquiry into the aims of science. Westport, Connecticut: Greenwood Press.

Weiskopf, D. A. (2011). The functional unity of special science kinds. The British Journal for the Philosophy of Science, 62(2), 233-258.

Wilkerson, T. E. (1995). Natural Kinds, Avebury Series in Philosophy. Aldershot: Avebury.

Wilson, R. A., Barker, M. J., \& Brigandt, I. (2007). When traditional essentialism fails: biological natural kinds. Philosophical Topics, 35(1/2), 189-215. 
Anouk Barberousse is professor of philosophy of science at Sorbonne Université (Paris). Her main research domains are philosophy of physics, philosophy of probability, epistemology of scientific images, and philosophy of scientific expertise. One of her representative publications is "Empirical agreement in model validation", with Julie Jebeile, Studies in History and Philosophy of Science Part A 56, 168-174 (2016).

Address: UFR de Philosophie, Faculté des Lettres, Sorbonne Université, 1 rue Victor Cousin, 75005 Paris, France. E-mail: anouk.barberousse@paris-sorbonne.fr

Françoise Longr is professor of philosophy of science at Université de Strasbourg and member of the Institut d'histoire et de philosophie des sciences et des techniques de Paris. One of her representative publications is "Do we need two notions of Natural Kind to account for the history of 'jade" "? Synthese 195, 1459-1486 (2018).

Address: Faculté de Philosophie, Université de Strasbourg, 7 rue de I'Université, 67000 Strasbourg, France \& Institut d'histoire et de philosophie des sciences et des techniques (IHPST), 13 rue du Four, 75006 Paris, France. E-mail: fralongy@gmail.com

Francesca Merlin is permanent researcher at CNRS (France). Her research focuses on central concepts in biology such as chance and probability, inheritance and epigenetics and, more recently, on the question of scientific pluralism in the study of environmentally induced diseases. One of her representative publications is "Epigenetics: A way to bridge the gap between biological fields", with Antonine Nicoglou, Studies in History and Philosophy of Biological and Biomedical Sciences Part C 66, 73-82 (2017).

Address: Institut d'histoire et de philosophie des sciences et des techniques (IHPST), CNRS \& Université Paris 1 Panthéon-Sorbonne, 13 rue du Four, 75006 Paris, France. E-mail: francesca.merlin@univparis1.fr / ORCID: 0000-0002-2335-3429

Stéphanie Ruphy is professor of philosophy of science at the Ecole normale supérieure - Université PSL. Her latest book is Scientific Pluralism Reconsidered, Pittsburgh University Press (2016).

Address: Département de Philosophie, Ecole normale supérieure - Université PSL, 45 rue d’Ulm, 75005 Paris, France. E-mail: stephanie.ruphy@ens.psl.eu / Website: www.stephanieruphy.com / ORCID: 0000-0002-7078-5136 